\title{
Recherches en soufflerie sur l'action relative de la houle et du vent
}

\section{Wind tunnel research on relative wave and wind action}

\author{
J HAN LARRAS \\ INGÉNIEUn GÉNÉRAL DES PONTS ET CHAUSSÉES, \\ PROFESSEUR A I'E.N.S.E.E.H. DE TOULOUSE
}

PAR

ET

\author{
AldBert CLARTA \\ CHEF DE LA SECTION AÉRODYNAMIOUE \\ DE L'INSTTTUT DE MÉCANIQUe Des FLUIDES DE TOULOUSE
}

\begin{abstract}
Les auteurs ont étudié l'action d'un vent parfaitement horizontal de 11,1 à $37,4 \mathrm{~m} / \mathrm{s}$ sur la maquette en bois verni d'une houle de $0,20 \mathrm{~m}$ de longueur d'onde et de 1,7 ou 3,3 ou 5,0 pour cent de cambrure.

Cette maquette comportait cinq ondulations de houle successives, les mesures n'ont eu lieu qu'all droit de l'ondulation centrale, et l'on a pris toutes précuntions spéciales pour éviter les dissymétries d'écoulement qui semblent avoir faussé les résultats d'expériences antérieures sur le mème sujet.

Les essais ont montré qu'il y a répartition presque symétrique des pressions du vent sur la surface libre de part et d'autre des crêtes, et que ces pressions sont proportionnelles au carré de la vitesse de l'air au point considéré (le coefficient de proportionnalité étant tel qu'il $y$ ait une surpression sur les crêtes avec une dépres. sion dans les creux, et que la courbe de répartition des surpressions soit légèrement plus pointue que la courbe de répartition des dépressions).
\end{abstract}

I. - La répartition des pressions du vent relatif sur la surface instantanée, momentanément figée, de la houle cylindrique simple, a fait l'objet de recherches expérimentales de Motzfeld dans un courant d'air à la soufflerie de l'Institut Kaiser-Wilhelm de Gottingen en 1937 (1), et de Thijsse dans un courant d'eau rectiligne uniforme au Laboratoire d'Hydraulique de Delft en 1952 (2).

Nous avons étudié la question à notre tour par des mesures en soufflerie à l'Ecole Nationale Supérieure d'Electrotechnique, d'Flectronique el

(1) Z. Angew. Math. U. Mech., cahier 4, août 1937.

(2) Gravity Waves, Circ. 521, Nat. Bureau of Standards, Depart. of Commerce, U.S.A., 1952.

\begin{abstract}
The authors have been studying the action of a perfectly horizontal wind at speeds ranging from $11.1 \mathrm{~m} / \mathrm{s}$ to $37.4 \mathrm{~m} / \mathrm{s}$ on a varnished wood model of waves having a wave length of $0.20 \mathrm{~m}$ and steepnesses of $1.7 \%, 3.3 \%$ or $5 \%$.

The model featured five successive wave undulations. The measurements were only taken at the central undulation, whereby all the necessary precautions were taken to prevent the occurence of unsymmetrical flow conditions, which seemed to have been a source of error in previous experiments on the same problem.

The tests showed that the wind pressure distribution over the free surfaces to either side of the crests is almost symmetrical, and that the wind pressures are proportional to the square of the air velocity at the considered point (the proportionality factor is such that there is a positive pressure on the cres/s and $a$ depression in the hollows, and that the positive pressure distribution curve has a slightly more pronounced peal than the corresponding curve for the depressions).
\end{abstract}

d'Hydraulique de Toulouse en 1959, et la présente note en constitue l'exposé.

II. - Nous avons représenté la surface momentanément figée de Ja houle par un modile en bois verni de $1,00 \mathrm{~m}$ de long dans la veine d'une soufflerie de $80 \mathrm{ch}$ de $2,40 \mathrm{~m}$ de diametre,
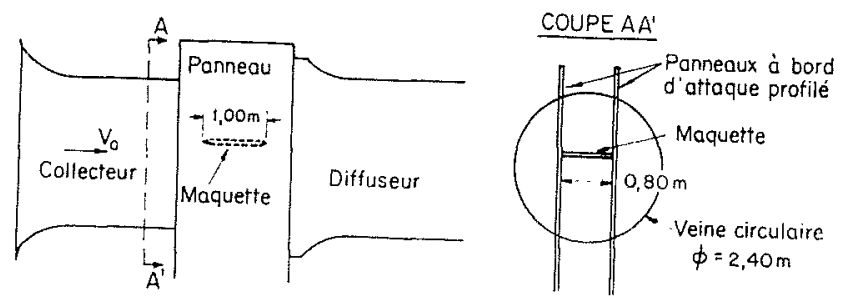

FIG. 1 
entre deux joues de guidage distantes de $0,80 \mathrm{~m}$ ices joues servant à maintenir un écoulement plan parallèle sur modèle en lui donnant les propriétés de l'allongement infini).

Le modele comportait cinq ondulations identiques soigneusement vernissées sur les deux faces, el on présentait l'ensemble au vent sous incidence nulle, de façon qu'il n'y ait pas de possibilités d'erreur appréciable dans les mesures de pression le long de londulation centrale.

La surface du modèle avait la forme d'une trochoïde, comme la surface libre de la houle cylindrique simple parfaitement pure (3), avec des ondulations de $0,20 \mathrm{~m}$ de longueur d'onde $(2 \mathrm{~L}=0,20 \mathrm{~m}$ ) et de 1,7 ou 3,3 ou $5 \%$ de cambrure suivant la série d'essais considérée.

Le corps du modèle comportait vingt et une prises de pression d'un bout à l'autre de l'ondulation centrale, à raison d'une par centimètre le long d'une bande métallique de $3 \mathrm{~cm}$ de largo encastrée dans le bois et parfaitement arasée par rapport à lui.

Nous avons effectué chaque série d'essais pour six vitesses de vent (relatif) allant de 11,1 à $37,4 \mathrm{~m} / \mathrm{s}$, et nous avons contrôlé le bon écoulement de l'air le long de la maquette au moyen de quatre prises supplémentaires situées de part et d'autre de l'ondulation centrale (4).

1II. - Nos essais nous onl montré qu'il y avait répartition presque symétrique des pressions du vent sur la surface libre de part et d'autre des crêtes alors qu'il y avait répartition dissymétrique, malgré la symétrie de la surface libre, dans les essais de Motzfeld et de Thijsse (5). Mais la dissymétric constatée par ces deux auteurs provenait peut-être de la trop lorte cambrure de la houle étudiée (6) et plus probablement d'unc fégère incidence du modèle jar rapport à la direction générale du vent à l'infini (faute de l'ẻgalisation parfaile des débils provenant de l'em-

(3) Il n'existe à vrai dire pas de houle cylindrique simple parfaitement pure en presence du vent, mais celui-ci ne modifie pas la forme de la surface libre d'unc facon qui influe notablement sur les conditions d'ćcoulement, et sur la répartition des pressions, du vent en surface.

(4) $2^{\theta}$ creux, $2^{*}$ crête, $4^{*}$ crête, $5^{\mathrm{e}}$ creux (en partant de l'amont, et en considérant le bord d'attaque du modèle comme le $1^{\text {er }}$ creux).

(5) La dissymétrie de la répartition des pressions correspond a lit résistance des ondulations de la surface libre à lat propagation du vent. Cette resistance inévitable était done extrêmencent faible dans le cas de nos essais.

(6) $2 \mathrm{~L}=0,15 \mathrm{~m}, 2 \mathrm{~h}=14,5 \mathrm{~mm}$. Cambrure voisine de $10 \%$, supérieure aux cambrures usuelles des lames de tempête.

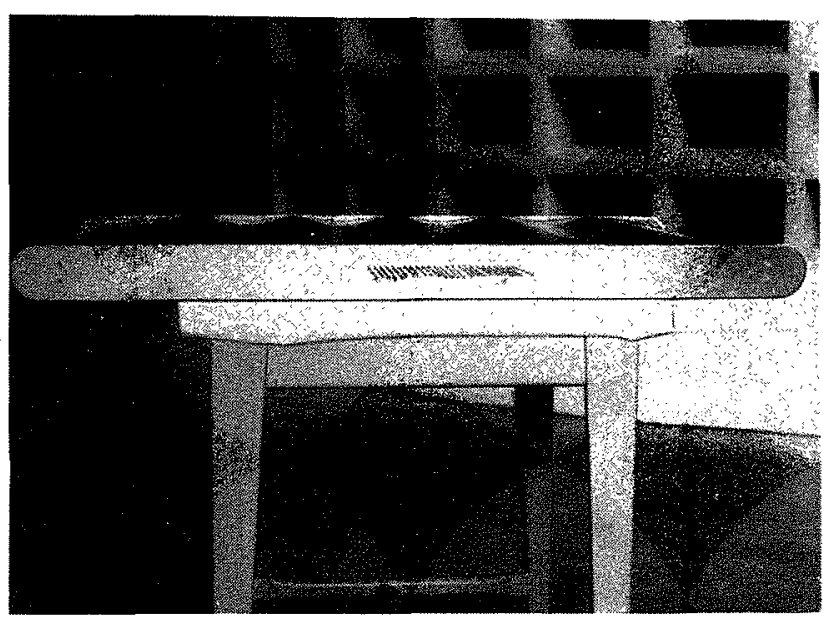

FIG. 2. - Vue du profil de la maquette cu avant du diffuseur.

ploi d'une maquette image symétrique de la maquetle de mesure).

Nos essais nous ont nontré, d'autre part, qu'il existe de plus fortes pressions sur les crêtes que de dépressions dans les creux. Ces résultats s'apparentent à ceux de Motzfeld et de Thijsse sur le plan qualitatif, mais ils s'en écartent sur le plan quantitatif (sous la forme, principalement, d'un déplacement du zéro donl nous parlerons plus loin) pour les mêmes raisons sans doute que celles déjà dites pour la dissymétrie des pressions de part et d'autre des crêtes.

Nos essais nous ont enfin monlré que la pression du vent sur la surface libre de la houle momentanément figée vaut ià quelques pour cent près) :

$$
p=\mathbf{K} \frac{\pi}{2 g} \cdot \mathbf{V}_{10}
$$

avec :

$$
K=\left(\frac{V}{V_{0}}\right)^{2}
$$

en désignant par :

$Y_{0}$ la vilesse à l'infini amont;

$V$ la vitesse au point considéré;

क le poids volumique de l'air.

Le coefficient $K$ ne difrère pratiquement pas de 1,12 à la distance $0,29 \times 2 \mathrm{I}$ de part et d'autre des creux, quelle que soit la cambrure (du moins pour les trois cambrures étudices)

La courbe de répartition des surpressions esl légèrement plus pointue que la courbe de répar- 
I. - CambruRE 1,67\%

\begin{tabular}{|c|c|c|c|}
\hline \multirow{2}{*}{$\begin{array}{l}\text { Distance } \\
\text { au creux } \\
\text { amont } \\
2 L \times\end{array}$} & \multicolumn{2}{|c|}{$\begin{array}{l}\text { Valeur de } \mathrm{K} \\
\text { pour } \mathrm{V}(\mathrm{m} / \mathrm{s})=\end{array}$} & \multirow{2}{*}{ Moyenne } \\
\hline & 17 & 39 & \\
\hline 0 & 1 & 1 & 1 \\
\hline 0,05 & 1,019 & 1,02 & 1,020 \\
\hline 0,10 & 1,025 & 1,027 & 1,026 \\
\hline 0,15 & 1,03 & 1,04 & 1,035 \\
\hline 0,20 & 1,06 & 1,055 & 1,0575 \\
\hline 0,25 & 1,085 & 1,09 & 1,0875 \\
\hline 0,30 & 1,115 & 1,11 & 1,1125 \\
\hline 0,35 & 1,17 & 1,15 & 1,160 \\
\hline 0,40 & 1,19 & 1,18 & 1,185 \\
\hline 0,45 & 1,194 & 1,205 & 1,204 \\
\hline 0,50 & 1,2 & 1,215 & 1,207 \\
\hline 0,55 & 1,195 & 1,2 & 1,1975 \\
\hline 0,60 & 1,17 & 1,18 & 1,175 \\
\hline 0,65 & 1,15 & 1,17 & 1,16 \\
\hline 0,70 & 1,135 & 1,145 & 1,14 \\
\hline 0,75 & 1,11 & 1,11 & 1,11 \\
\hline 0,80 & 1,06 & 1,057 & 1,0585 \\
\hline 0,85 & 1,03 & 1,035 & 1,0325 \\
\hline 0,90 & 1,02 & 1,02 & 1,020 \\
\hline 0,95 & 1,015 & 1,018 & 1,0165 \\
\hline 1,00 & 1 & 1,005 & 1,002 \\
\hline
\end{tabular}

II. - Cambrure 3,33, \%

\begin{tabular}{|c|c|c|c|c|}
\hline \multirow{2}{*}{$\begin{array}{c}\text { Distance } \\
\text { au ereux } \\
\text { amont } \\
2 \mathrm{~L} \times\end{array}$} & \multicolumn{3}{|c|}{ Valeur de $\mathrm{K}$ pour $\mathrm{V}(\mathrm{m} / \mathrm{s})=$} & \multirow{2}{*}{ Moyenne } \\
\hline & 14,75 & 27,30 & 38,60 & \\
\hline 0 & 0,921 & 0,915 & 0,92 & 0,919 \\
\hline 0,05 & 0,921 & 0,916 & 0,905 & 0,914 \\
\hline 0,10 & 0,948 & 0,942 & 0,935 & 0,942 \\
\hline 0,15 & 0,967 & 0,972 & 0,962 & 0,967 \\
\hline 0,20 & 1,026 & 1,02 & 1,015 & 1,02 \\
\hline 0,25 & 1,106 & 1,102 & 1,106 & 1,089 \\
\hline 0,30 & 1,153 & 1,142 & 1,15 & 1,148 \\
\hline 0,35 & 1,187 & 1,185 & 1,173 & 1,182 \\
\hline 0,40 & 1,247 & 1,251 & 1,25 & 1,249 \\
\hline 0,45 & 1,24 & 1,24 & 1,235 & 1,238 \\
\hline 0,50 & 1,267 & 1,285 & 1,285 & 1,279 \\
\hline 0,55 & 1,24 & 1,24 & 1,24 & 1,24 \\
\hline 0,60 & 1,226 & 1,24 & 1,23 & 1,232 \\
\hline 0,65 & 1,187 & 1,21 & 1,20 & 1,199 \\
\hline 0,70 & 1,146 & 1,152 & 1,147 & 1,148 \\
\hline 0,75 & 1,092 & 1,1 & 1,1 & 1,097 \\
\hline 0,80 & 1,04 & 1,055 & $\mathrm{I}, 048$ & 1,048 \\
\hline 0,85 & 0,99 & 0,98 & 0,98 & 0,983 \\
\hline 0,90 & 0,948 & 0,945 & 0,942 & 0,945 \\
\hline 0,95 & 0,923 & 0,93 & 0,92 & 0,924 \\
\hline 1,00 & 0,915 & 0,915 & 0,92 & 0,917 \\
\hline
\end{tabular}

III. - CambruRE $5 \%$

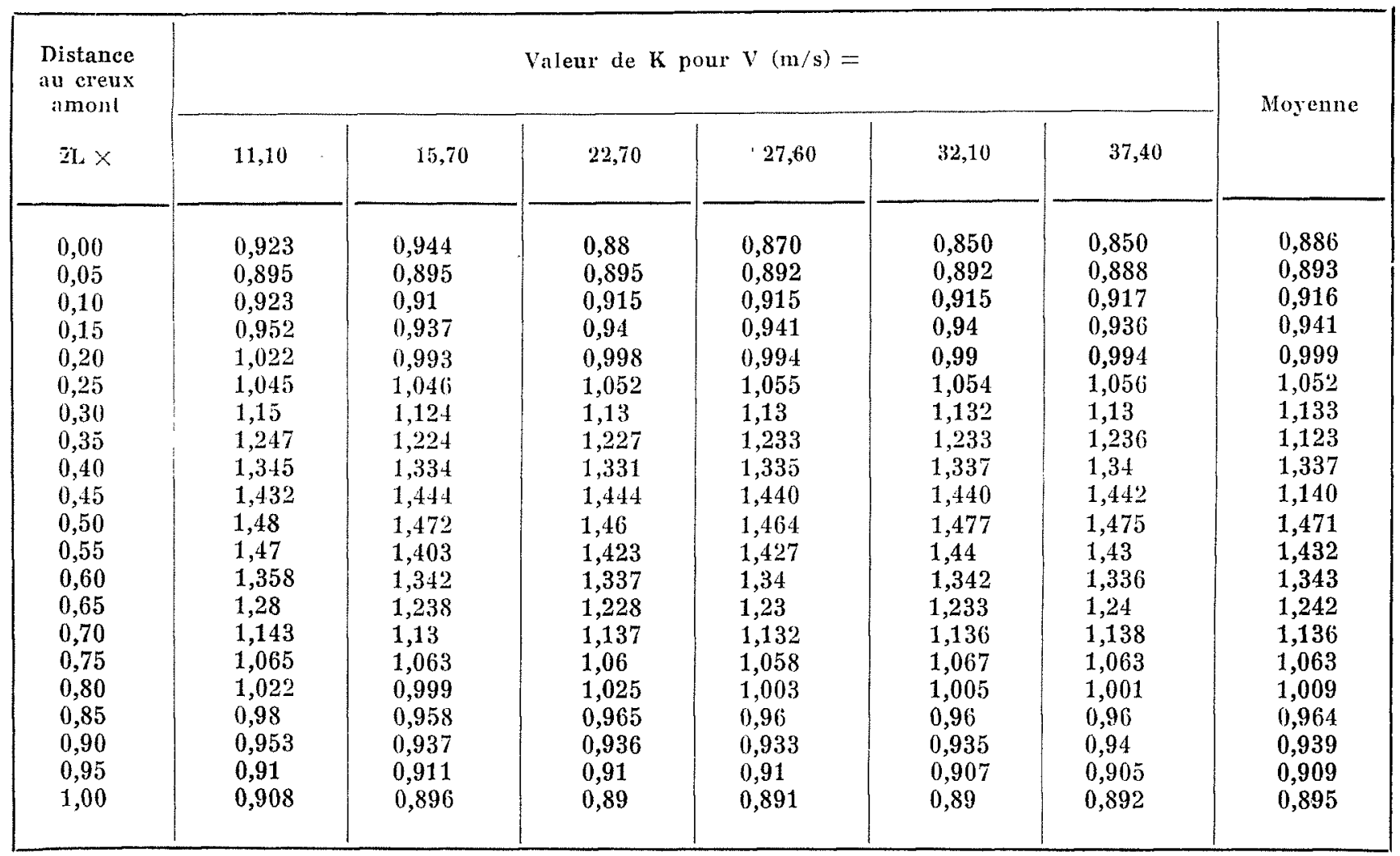




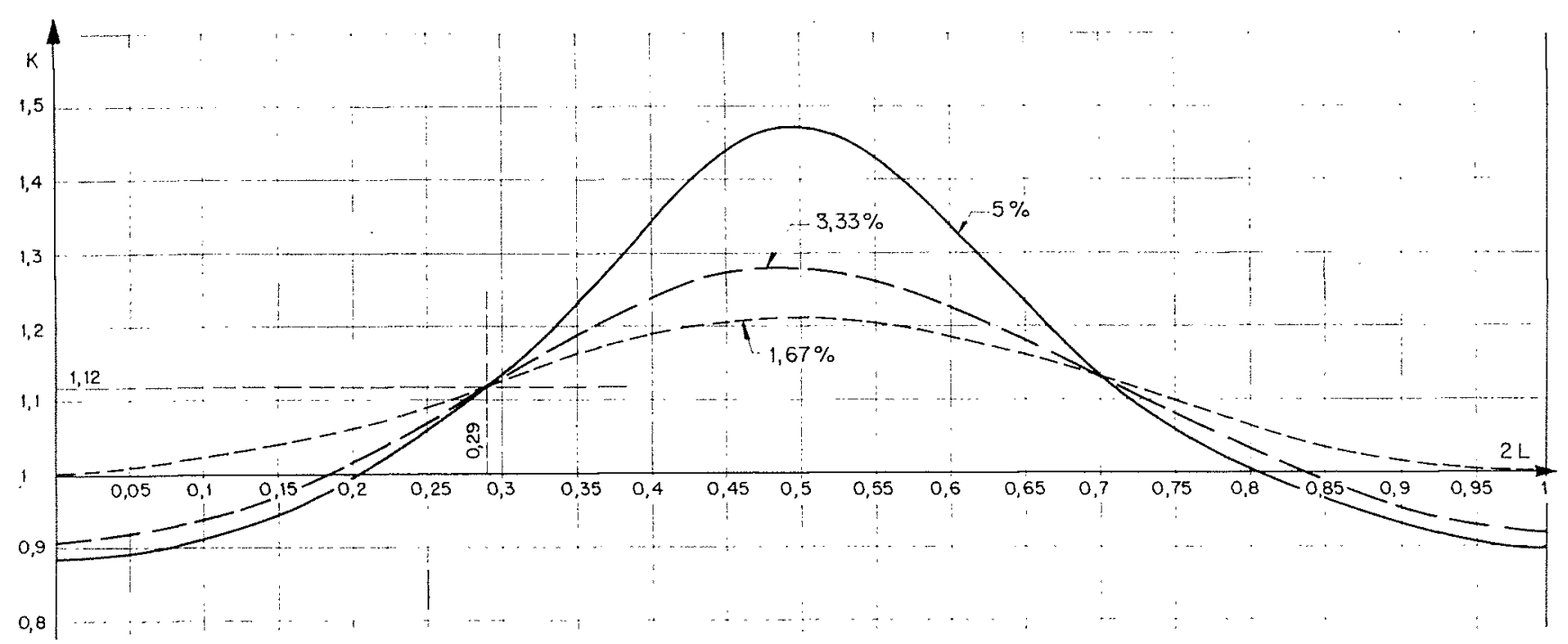

FIG. 3

lilion des dépressions, comme il ressort des résultats suivants :

\begin{tabular}{|c|c|c|c|}
\hline \multirow{2}{*}{$\begin{array}{c}\text { Distance } \\
\text { de la prise } \\
\text { de pression } \\
\text { au creux nmont }\end{array}$} & \multicolumn{3}{|c|}{ Valeul's de $\mathrm{K}$ pour une cambiure de } \\
\hline & $1,67 \%$ & $3.33 \%$ & $5 \%$ \\
\hline $\begin{array}{c}x=0,0 \times 2 \mathrm{~L} \\
(\text { creux }) \\
0,1 \times 2 \mathrm{~L} \\
0,2 \times 2 \mathrm{~L} \\
0,3 \times 2 \mathrm{~L} \\
0,4 \times 2 \mathrm{~L} \\
0,5 \times 2 \mathrm{~L} \\
\text { (crete) }\end{array}$ & $\begin{array}{l}1,00 \\
1,02 \\
1,06 \\
1,13 \\
1,18\end{array}$ & $\begin{array}{l}0,92 \\
0,94 \\
1,03 \\
1,15 \\
1,24\end{array}$ & $\begin{array}{l}0,89 \\
0,92 \\
1,00 \\
1,13 \\
1,34\end{array}$ \\
\hline
\end{tabular}

Et l'on peut considérer l'écart total de pression entre les crêtes et les creux comme pratiquement proportionnel à la cambrure $\gamma$ de la houle :

$$
\begin{aligned}
& \gamma=1,67 \% \quad \Delta \mathrm{K}=0,21 \quad \frac{\Delta \mathrm{K}}{\gamma}=0,126 \\
& \begin{array}{lll}
3,33 & 0,36 \quad 0,111
\end{array} \\
& \begin{array}{lll}
5,00 & \mathbf{0 , 5 8} & 0,116
\end{array}
\end{aligned}
$$

IV. - L'ensemble des résultats précédents nous paraît devoir présenter le plus vif intérêl pour l'étude mathématique de la formation el de l'évolution de la houle sous l'action du vent.

I a présence sur les crêtes d'une pression dýnamique supérieure à la pression correspondante à l'infini amont, et inférieure ou égale dans les creux, donne en effet à penser que le vent tend à augmenter naturellement l'amplitude de la houle lorsqu'il souffle de facon parfaitement parallèle à l'horizon sans décollement sur le profil (7).

Ia symétrie presque absolue de la répartition des pressions dynamiques sur la surface libre de part et d'autre des crêtes semble montrer en outre que les ondulations des vagues prísentent peu de résistance à la propagation du vent dans les mêmes conditions.

(7) Il semblerait $y$ avoir, tout an contraire, tendance à réduction d'amplitude de la houle sous l'action du vent, en cas de décollements sur le profll, comme il s'en produit par mer très creuse pour des cambirures supérieures aux cambrures usuelles de tempête, seuless considérées dans nos essais. 